\title{
EDITORIAL / ÉDITORIAL
}

\section{Health Surveillance: Changing Needs, Constant Function}

\section{Don Wigle and David Mowat ${ }^{2}$}

\begin{abstract}
"Would you tell me please, which way I ought to go from here?" "That depends a good deal on where you want to get to," said the Cat. "I don't much care where_" said Alice. "Then it doesn't matter which way you go," said the Cat.
\end{abstract}

- Alice's Adventures in Wonderland, Lewis Carroll

In a landmark report, "The Future of Public Health," the Institute of Medicine defined the core public health functions to be assessment, assurance and advocacy. ${ }^{1}$ Health surveillance is an essential tool for the achievement of these core public health functions, particularly at a time of rapid change driven by fiscal restraint. Health surveillance helps those responsible for public health change management to know where they are, to assess needs and priorities and to monitor progress toward public health goals.

About 50 years ago, Dr. Alexander Langmuir joined the then recently formed US Communicable Disease Center (CDC). He launched the first-ever disease surveillance program and confirmed his suspicion that malaria, on which CDC spent the largest portion of its budget at that time, had long since disappeared from the United States. Since that time, disease surveillance has grown to be CDC's core function in support of public health practice.

In its recent report "An Integrated National Health Surveillance Network for Canada," ${ }^{2}$ the Federal-ProvincialTerritorial (FPT) Surveillance Integration Design Team defined health surveillance as follows:

"The tracking and forecasting of any health event or health determinant through the ongoing collection of data, the integration, analysis and

1. Bureau of Operations, Planning and Policy, Laboratory Centre for Disease Control, Health Protection Branch, Health Canada

2. Surveillance Transition, Health Protection Branch, Health Canada interpretation of those data into surveillance products and the dissemination of such products to those who need to know. Surveillance products are produced for a predetermined public health purpose or policy objective. In order to be considered health surveillance, all of the above activities must be carried out."

The single characteristic that makes health surveillance distinct from other health information activities is the requirement for a direct link to a public health purpose or policy objective. In general, it entails organized, population-based data collection on a frequent or continuous basis. Timeliness of surveillance products is usually a requirement. However, health surveillance is a component of the broader field of health information and is highly dependent on health information standards, practices and technologies as well as the legal and ethical constraints of using information about individuals.

If we accept that health surveillance is a measurement tool that serves public health objectives, then it follows that the scope of health surveillance and the methods used must change to meet changing needs. In fact, this has occurred to a considerable degree. Whereas health surveillance was once limited to monitoring the occurrence of infectious diseases, the scope has expanded to include non-infectious diseases, injuries, early detection of emerging and re-emerging diseases and risk behaviours. Data collection methodologies have evolved from paper-based reports to electronically linked administrative health record systems, population surveys and internet-based technologies. Analytic methods have progressed from simple counts and rates to modelling and forecasting.

The Krever Commission Report ${ }^{3}$ was the final report of the Commission of Inquiry on the Blood System in Canada. Justice Krever made several recommendations with implications for health surveillance in Canada including:

1) In matters of public health and safety, there is often a need to act in the absence of complete information; the more severe the potential effect, the lower the threshold should be for taking action. By providing information on persons with adverse health outcomes (numbers affected, severity of health effects), health surveillance provides a basis for taking action on factors associated with current health outcomes.

2) Post-marketing surveillance is needed to evaluate the appropriate use of and access to essential products and for early detection of adverse health effects.

3) Surveillance information is needed to inform the public about the performance of the health system so they can provide informed contributions to decision making.

4) Surveillance of the public health system per se is needed to evaluate the adequacy of resources available to implement essential public health policies and programs.

Although the scope of the Krever Inquiry was limited to infectious diseases transmissible through blood and blood products, the principles apply equally to the full span of public health services including:

1) Public health policy and risk management: Surveillance identifies health needs and progress in health protection and health promotion.

2) The immediate control of disease: Timeliness is vitally important in the management of infectious diseases which have the potential to rapidly place many Canadians at risk of serious illness or death. Early detection of infectious disease outbreaks is needed to trigger rapid implementation of control measures and may serve specific legislated mandates (e.g., food safety). 
3) Monitoring and forecasting trends of health problems and their determinants to provide early warning of significant changes (e.g., teen smoking) and to identify high-risk groups (e.g., groups at risk for HIV/AIDS).

There are important secondary uses of surveillance information such as hypothesis generation, population research (e.g., on health services, program evaluation) and the provision of basic information on health status indicators for the public.

There are major gaps in Canada's health surveillance systems. Although we have quite good information on mortality from many diseases and injuries, we only have national population-based incidence data for cancer and HIV/AIDS. It is hard to believe but we have little or no incidence data for most diseases including coronary heart disease, stroke, cirrhosis, diabetes, injuries and other preventable health conditions. Even for cancer, we lack information on extent of disease at diagnosis which is needed to evaluate the impact of cancer screening and treatment programs.

In the area of risk factors and health determinants, we have inadequate or no surveillance systems for drinking water quality, air quality, risk behaviours (although there are developments in this field), nutritional status, physical fitness and access to preventive health services. The absence of basic population-based incidence and exposure information means that we seldom have information on the disease burden attributable to a given risk factor, a very important indicator needed to assess public health priorities. We do not yet have readily accessible risk factor and disease incidence, prevalence and mortality data stratified by income, education and other broad health determinants. Surveillance requires a commitment to collect standardized, comparable measures over time - even this basic requirement is rarely met in practice.

As Canada enters the new millennium, there are other unmet surveillance needs including comprehensive surveillance information on target populations (e.g., low-income groups, children, women, Aboriginals, refugees/migrants and seniors), health consequences of natural disasters, climate extremes and other potential effects of global climate change, hazards related to consumer and commercial products (post-marketing surveillance) and occupational safety and health.

The FPT Surveillance Integration Design Team noted a number of gaps related to specific diseases, but concluded that a more pressing need was to address those needs that many surveillance systems had in common. These include:

1) Common protocols and standards for access to, interpretation and release of data, and for the protection of privacy.

2) Improving access to existing information - inventories of existing databases, metadata and expertise, together with a portal or "single window" means of access, using new technology, particularly to facilitate timely access to data.

3) Developing and adopting standards for the classification of diseases, exposures and other health events, data base elements, and for informatics.

4) Developing and sharing innovative electronic means of accessing, integrating, analyzing, presenting and disseminating information.

5) Strengthening the human resources and skills available to perform surveillance across Canada.

It is proposed that federal, provincial, local/regional, NGO and academic partners can improve the capacity to undertake surveillance through participation in the proposed network for health surveillance in Canada.

It has been said that, "What gets measured gets done." If one accepts this, then the surveillance gaps and deficiencies alluded to above imply that Canadians are likely not receiving optimal interventions to reduce their risk of preventable illness and premature death. There are, however, signs of progress. The Canadian Coalition on Cancer Surveillance arose from a National Cancer Institute of Canada workshop held at Kananaskis in November 1996. Cooperation between voluntary, professional, provincial and federal agencies led to agreement on needs and priorities for cancer surveillance in Canada and followup activities should achieve improved information such as basic staging data for newly diagnosed cancer patients. Similar activities are underway for cardiovascular disease and diabetes.

Health Canada has undertaken several novel proof-of-concept surveillance projects to test the feasibility of real-time surveillance systems and the strengthening of local/regional/national/global surveillance systems. The Roadmap project under development by Health Canada, Statistics Canada and the Canadian Institute for Health Information is designed to provide improved information on the performance of the health care system and the health status of Canadians. The main thrusts of Roadmap are to create person-oriented health care records, to expand the National Population Health Survey to subprovincial health regions, to improve or create various measures of health care utilization (including use of pharmaceuticals, home care, mental health, addictions, rehabilitation and primary care), to improve data standards and data sharing, strengthen disease registries, and to facilitate costing of health services by developing costing methodologies and mechanisms.

The discussion above has focussed on the need for surveillance to meet one of the core functions of public health, i.e., assessment. Surveillance is also important to achieve the other core functions, assurance and advocacy. Timely accurate surveillance data are essential to assess health needs and to justify the resources needed to provide assurance of effective health protection, health promotion and disease control programs. Such data are also needed by voluntary agencies in their advocacy role to "make the case" with the public and policy makers.

In meeting the challenges of improving health surveillance in Canada, it will be important to have focus and collaboration. Focus is needed to concentrate limited resources on questions of national public health importance and the needs of the major users of health surveillance products. Collaboration is essential for the efficient and effective improvement and sharing of health data. Let us hope that we in the public health surveillance field are up to this challenge. 


\section{Surveillance de la santé : des besoins changeants, une fonction constante}

\author{
Don Wigle et David Mowat ${ }^{2}$
}

"Si tu ne sais pas où tu vas, n'importe quel chemin t'y mènera "

- Alice au pays des merveilles, Lewis Carroll

Dans un rapport qui a fait date, "The Future of Public Health, " l'Institute of Medicine a défini les principales fonctions de la santé publique comme étant l'évaluation, l'élaboration de politiques et la promotion des intérêts. ${ }^{1}$ La surveillance de la santé est un outil essentiel pour remplir ces fonctions de base relatives à la santé publique, surtout à une époque de changement rapide imposé par des restrictions budgétaires. La surveillance de la santé permet à ceux qui sont responsables de la gestion de la santé publique de savoir où ils en sont, d'évaluer les besoins et les priorités et de suivre les progrès pour atteindre les objectifs de santé publique.

Il y a une cinquantaine d'années, le $\mathrm{D}^{\mathrm{r}}$ Alexander Langmuir est entré au service du US Communicable Disease Center (CDC) qui venait tout juste d'être créé. Il a alors lancé le tout premier programme de surveillance des maladies qui a confirmé ce dont on se doutait, à savoir que le paludisme, auquel le CDC consacrait alors la plus importante partie de son budget, avait depuis longtemps disparu des États-Unis. Depuis cette époque, la surveillance des maladies a pris de l'expansion pour devenir la principale fonction du CDC pour venir aider la santé publique.

Dans un rapport récent intitulé "un Réseau national de surveillance de la santé intégré pour le Canada, " ${ }^{2}$ l'Équipe fédérale-provinciale-territoriale de conception de l'intégration a défini la surveillance de la santé de la façon suivante :

"Le suivi et l'évaluation de tout

événement ou de tout facteur détermi-

1. Bureau des opérations, de la planification et des politiques, Laboratoire de lutte contre la maladie, Direction générale de la protection de la santé, Santé Canada

2. Transition de surveillance, Direction générale de la protection de la santé, Santé Canada nant relatifs à la santé par le biais de la collecte continuelle de données, ainsi que l'intégration, l'analyse et l'interprétation de ces données par des produits de surveillance. En plus, on compte la distribution des produits de surveillance qui en résultent à ceux qui ont besoin de connaître la situation. Les produits de surveillance sont conçus à des fins prédéterminées de santé publique ou d'objectif politique. Afin d'être considérée comme la surveillance de la santé (sic), toutes les activités notées doivent s'accomplir. "

L'unique caractéristique qui distingue la surveillance de la santé de toute autre activité visant à recueillir de l'information sur la santé est l'exigence d'un lien direct avec une raison ou un objectif de politique de santé publique. En général, cette surveillance oblige de recueillir des données démographiques de manière structurée et de façon fréquente ou régulière. La disponibilité en temps opportun des données de surveillance est généralement obligatoire. Toutefois, la surveillance de la santé fait partie du domaine plus large de l'information sur la santé et à ce titre dépend beaucoup des normes, des pratiques et des technologies propres à cette information ainsi que des contraintes éthiques et légales rattachées à l'utilisation des renseignements concernant les individus.

Si l'on reconnaît que la surveillance de la santé est un outil de mesure au service des que la portée de cette surveillance et les méthodes utilisées doivent évoluer pour répondre aux besoins changeants. En fait, c'est ce qui s'est passé dans une très large mesure. Alors que la surveillance de la santé était autrefois cantonnée au suivi des cas de maladies infectieuses, elle s'est étendue aux maladies non infectieuses, aux objectifs de la santé publique, il s'ensuit blessures, à la détection précoce des maladies émergentes et ré-émergentes ainsi qu'aux comportements à risque. Après avoir eu recours aux rapports écrits, les méthodes de collecte des données sont passées aux systèmes d'archivage électronique des dossiers médicaux, aux enquêtes sur la population et aux technologies utilisant Internet. Les méthodes d'analyse ont pour leur part aussi évolué, et de simples taux et décomptes sont passées à la modélisation et aux prévisions.

Le Rapport de la Commission Krever $^{3}$ a été le rapport final de la Commission d'enquête sur le système d'approvisionnement en sang au Canada. Le juge Krever a fait plusieurs recommandations ayant des implications pour la surveillance de la santé au Canada dont :

1) En matière de santé et sécurité du public, il faut parfois agir sans disposer de toute l'information nécessaire; plus le risque potentiel est élevé, plus bas doit se situer le seuil à partir duquel agir. En fournissant des renseignements sur les personnes qui connaissent des réactions indésirables (nombre de personnes touchées, gravité des réactions indésirables), la surveillance de la santé est à la base des mesures à prendre pour agir sur les facteurs associés à ces réactions.

2) Il est nécessaire d'effectuer une surveillance postcommercialisation pour évaluer la bonne utilisation et l'accès aux produits essentiels de la surveillance ainsi que pour détecter rapidement les réactions indésirables.

3) Les données de la surveillance sont nécessaires pour informer le public du rendement du système de santé pour qu'il puisse en retour participer de façon informée à la prise de décisions.

4) Il est nécessaire de surveiller le système de santé publique en soi pour voir s'il y a suffisamment de ressources pour mettre en oeuvre ses programmes et ses politiques.

Bien que la portée de l'enquête Krever ait été limitée aux maladies infectieuses transmissibles par le sang et les produits dérivés du sang, les principes s'appliquent également à toute la gamme des services de santé publique dont notamment :

...suite à la page 155 
the cost of the project. If this information was collected and stored in an information base in the school system, and could be readily retrieved, the time saving could result in a lower cost screening program.

Screening of high-risk groups and prevention of TB by means of chemoprophylaxis is an important component of $\mathrm{TB}$ control, and is especially important for the age group under 20 years due to the combined advantage of a longer lifespan free from TB disease and the very low risk of side effects of INH medication. Addressing the issues which decrease the effectiveness of screening and prevention programs would improve results and consequently increase the cost-effectiveness of the program.

\section{REFERENCES}

1. Screening for tuberculosis and tuberculous infection in high risk populations. $M M W R$ 1990;39(RR-8):1-7.

2. The use of preventive therapy for tuberculosis infection in the United States. $M M W R$ 1990;39(RR-8):9-12.

3. Fitzgerald JM, Gafni A. A cost effectiveness analysis of the routine use of Isoniazid prophylaxis in patients with a positive Mantoux skin test. Am Rev Respir Dis 1990;142:848-53.

4. Bass JB. Tuberculin test, preventive therapy, and elimination of tuberculosis. Am Rev Respir Dis 1990;141:812-13.

5. McKenna MT, McCray E, Onorato I. Tuberculosis among foreign-born persons in the United States. N Engl J Med 1995;332(16):1071-76.

6. Bleiker MA. The annual tuberculosis infection rate, the tuberculin survey and the tuberculin test. Bull Int Union Tuberc Lung Dis 1991;66:53-56.

7. Adhikari N, Menzies R. Community-based tuberculin screening in Montreal: A costoutcome description. Am J Public Health 1995;85:786-90.

8. Yuan L, Richardson E, Kendall P. Evaluations of a tuberculosis screening program for high-risk student in Toronto schools. CMAJ 1995;153(7):925-32.

Received: June 19, 1998

Accepted: December 17, 1998
Éditorial, suite de la page 151

1) La politique de santé publique et la gestion des risques : la surveillance doit permettre de déterminer les besoins de santé et les progrès dans la protection et la promotion de la santé.

2) La lutte immédiate contre la maladie : la vitesse d'intervention est d'une importance vitale dans la gestion des maladies infectieuses qui peuvent potentiellement faire courir des risques de maladie grave, voire de décès, à de nombreux Canadiens. La détection précoce des flambées de maladie infectieuse est indispensable pour déclencher rapidement l'application de mesures de lutte et peut répondre à des obligations légales spécifiques (par ex., la salubrité des aliments).

3) Le suivi et la prévision des problèmes de santé et de leurs déterminants pour tirer rapidement la sonnette d'alarme en cas de changements significatifs (par ex., le tabagisme des adolescents), et pour identifier les groupes à risque élevé (par ex., les groupes à risque pour le $\mathrm{VIH} /$ sida).

Les données de surveillance ont aussi des utilisations secondaires importantes, par exemple pour la formulation d'hypothèses, pour la recherche sur la population (par ex., sur les services de santé, sur l'évaluation des programmes) et pour donner au public une information de base sur les indicateurs de l'état de santé.

Les systèmes de surveillance de la santé au Canada présentent de grandes carences. Bien que nous disposions d'une bonne information sur la mortalité résultant d'un grand nombre de maladies et de blessures, nous n'avons que des données démographiques nationales sur l'incidence du cancer et sur celle du VIH/sida. Pour aussi incroyable que ce soit, nous n'avons que peu ou pas de données sur l'incidence de la plupart des maladies, notamment pour ce qui est des maladies coronariennes, des accidents cérébro-vasculaires, de la cirrhose, du diabète, des blessures et autres pathologies que l'on peut prévenir. Même dans le cas du cancer, nous manquons d'informations sur l'ampleur de la maladie à la pose du diagnostic, informations dont on a besoin pourtant pour évaluer l'impact du dépistage du cancer et des programmes de traitement.

S'agissant des facteurs de risque et des déterminants de la santé, les systèmes de surveillance sont insuffisants ou inexistants pour la qualité de l'eau potable, la qualité de l'air, les comportements à risque (même s'il y a des évolutions dans ce domaine), l'état nutritionnel, la forme physique et l'accès aux services de prévention. L'absence d'informations démographiques sur l'incidence et l'exposition aux maladies signifie que nous disposons rarement d'informations relatives au fardeau de la morbidité attribuable à un facteur de risque donné, un indicateur pourtant très important pour évaluer les priorités en matière de santé publique. Nous n'avons pas encore accès facilement aux données concernant les facteurs de risque, l'incidence, la prévalence et la mortalité propres à une maladie donnée ventilées en fonction du revenu, du niveau de scolarité et d'autres grands déterminants de la santé. La surveillance exige que l'on s'engage à recueillir des données normalisées et comparables entre elles sur une longue durée; dans la pratique, même cette exigence fondamentale n'est pas satisfaite.

Alors que le Canada s'apprête à entrer dans le nouveau millénaire, il y a d'autres besoins de surveillance qui ne sont pas satisfaits, notamment des données complètes sur des populations cibles (par ex., les groupes à faible revenu, les enfants, les femmes, les Autochtones, les réfugiés/immigrants et les personnes âgées), sur les conséquences des catastrophes naturelles sur la santé, sur les variations climatiques extrêmes et autres effets potentiels du changement climatique mondial, sur les dangers associés aux produits de consommation et commerciaux (surveillance postcommercialisation) et sur la santé et la sécurité au travail.

L'Équipe fédérale-provinciale-territoriale de conception de l'intégration a constaté plusieurs carences s'agissant de maladies spécifiques, mais en est arrivée à la conclusion qu'il était beaucoup plus urgent de s'attaquer aux besoins communs à de nombreux systèmes de surveillance. Il s'agit notamment :

...suite à la page 180 
dence in Canada and its regions, 1969 to 1988. Ottawa: Canadian Council of Cancer Registries, 1993;16-21.

9. Le ND, Marrett LD, Robson DL, et al. Canadian Cancer Incidence Atlas, Vol. 1. Ottawa: Health Canada, 1995;20-24.

10. Horwich A, Malcolm DM, Hendry WF. Testicular tumours, In: Peckham M, Pinedo H, Veronesi U (Eds.), Oxford Textbook of Oncology Vol. 2, Oxford: Oxford University Press, 1995; $1407-14$

11. Clayton D, Schifflers E. Models for temporal variation in cancer rates, I: Age-period and Agecohort models. Stat Med 1987;6:449-67.

12. Clayton D, Schifflers E. Models for temporal variation in cancer rates, II: Age-period-cohort models. Stat Med 1987;6:469-81.

13. SAS Institute Inc., SAS Technical Report P-243, SAS/STAT Software: The GENMOD Procedure, Release 6.09, Cary, NC: SAS Institute Inc., 1993; 1-47.

14. Holford TR. Understanding the effects of age, period, and cohort on incidence and mortality rates. Annu Rev Public Health 1991;12:425-57.

15. Brown LM, Pottern LM, Hoover RN. Prenatal and perinatal risk factors for testicular cancer. Cancer Res 1986;46:4812-16.

16. Depue RH, Pike MC, Henderson BE. Estrogen exposure during gestation and risk of testicular cancer. J Natl Cancer Inst 1983;71:1151-55.

17. Gershman ST, Stolley PD. A case-control study of testicular cancer using Connecticut tumour registry data. Int J Epidemiol 1988;17:738-42.

18. Akre O, Ekbom A, Hsieh CC, et al. Testicular nonseminoma and seminoma in relation to perinatal characteristics. I Natl Cancer Inst 1996;88:883-89.

Received: April 9, 1998

Accepted: November 25, 1998

\section{Éditorial, suite de la page 155}

1) Des normes et des protocoles communs pour l'accès, l'interprétation et la publication de données, ainsi que pour la protection des renseignements personnels.

2) De l'amélioration de l'accès aux renseignements existants - inventaires des bases de données existantes, métadonnées et expertise, ainsi qu'un moyen d'accès de type "guichet unique » se servant des nouvelles technologies pour notamment accélérer l'accès aux données.

3) Du développement et de l'adoption de normes - pour la classification des maladies, des expositions et autres phénomènes de santé, des éléments des bases de données, ainsi que pour l'informatique.

4) De la mise au point et du partage de moyens électroniques innovateurs pour avoir accès, intégrer, analyser, présenter et disséminer l'information.

5) Du renforcement des ressources et des compétences humaines disponibles pour effectuer la surveillance partout au Canada.

On pense que les partenaires fédéraux, provinciaux, locaux et régionaux, les ONG et les établissements d'enseignement peuvent renforcer leurs moyens de surveillance en faisant partie du réseau de surveillance de la santé envisagé pour le Canada.

D'aucuns ont dit que "ce qui est mesurable est réalisable. "Si l'on accepte cette hypothèse, alors les carences et les déficiences de la surveillance que l'on vient de présenter ci-dessus signifient qu'il est fort probable que les Canadiens ne reçoivent pas les meilleures interventions pouvant réduire les risques qu'ils courent de contracter une maladie évitable ou de mourir prématurément. Toutefois, on constate des signes de progrès. La Canadian Coalition on Cancer Surveillance a été créé à la suite d'un atelier organisé par le National Cancer Institute of Canada à Kananaskis en novembre 1996. La coopération entre des organismes bénévoles, professionnels, provinciaux et fédéraux a débouché sur la reconnaissance des besoins et des priorités en ce qui concerne la surveillance du cancer au Canada et les mesures de suivi devraient se traduire par des améliorations de l'information comme les données de stadification de base pour les nouveaux cas diagnostiqués de cancer. Des activités analogues ont été lancées pour les maladies cardiovasculaires et pour le diabète.

Santé Canada a entrepris plusieurs projets de surveillance innovateurs de validation conceptuelle pour voir s'il est possible d'avoir des systèmes de surveillance en temps réel ainsi que pour tester le renforcement des systèmes de surveillance locaux, régionaux, nationaux et mondiaux. Le projet Roadmap en phase de développement par Santé Canada, Statistique Canada et l'Institut canadien d'information sur la santé est conçu de sorte à fournir de meilleures données sur le rendement du système de soins de santé ainsi que sur l'état de santé des Canadiens. Le projet Roadmap a pour principal objectif de créer des dossiers médicaux personnalisés, d'étendre l'Enquête nationale sur la santé de la population aux régions de santé infraprovinciales, d'améliorer ou de concevoir différentes mesures du recours aux soins de santé (y compris aux produits pharmaceutiques, aux soins à domicile, aux soins de santé mentale, aux soins de la toxicomanie, aux soins de rééducation et aux soins primaires), d'améliorer les normes des données et le partage de ces dernières, de perfectionner les registres des maladies, et de faciliter le calcul des coûts des soins de santé en mettant au point des mécanismes et des méthodes de détermination des coûts.

Les propos ci-dessus portaient essentiellement sur la nécessité pour la surveillance de satisfaire à l'une des principales fonctions de la santé publique, à savoir l'évaluation. La surveillance est également importante pour assurer d'autres fonctions essentielles, à savoir l'élaboration de politiques et la promotion des intérêts. Des données de surveillance exactes et disponibles au moment requis sont essentielles pour évaluer les besoins de santé et pour justifier les ressources nécessaires pour garantir des programmes efficaces de protection et de promotion de la santé ainsi que de lutte contre la maladie. Pareilles données sont

...suite à la page 185 


\section{REFERENCES}

1. Singer M, Anglin R, Song L, Linghofer L. Adolescents' exposure to violence and the association of psychological trauma. JAMA 1995:273:477-82.

2. Boney-MacCopy S, Finkelhor D. Psychosocial sequela of violent victimization in a national youth sample. J Consult Clin Psychol 1995;63:726-36.

3. Fitzpatrick KM, Boldizar JP. The prevalence and consequences of exposure to violence among African-American youth. J Am Acad Child Adolesc Psychiatry 1993;32(2):424-30.

4. Simmons R, Blyth D. Moving into Adolescence: The Impact of Pubertal Change and School Context. Hawthorne, NY: Aldine, 1987.

5. Dyson J. The effect of family violence on children's academic performance and behavior. J Natl Med Assoc 1990;82(1):17-22.

6. Freeman LN, Mokros H, Poznanski EO. Violent events reported by normal urban school-aged children: Characteristics and depression correlates. J Am Acad Child Adolesc Psychiatry 1993;32(2):419-23.

7. Richters JE, Martinez P. The NIMH community violence project I: Children as victims of and witnesses to violence. Psychiatry 1993;56:7-21.

8. Pynoos RS, Eth S. Children traumatized by witnessing acts of personal violence. In: Eth $S$, Pynoos R (Eds.), Post-traumatic Stress Disorder in Children. Washington, DC: American Psychiatric Press, 1985.

9. Rivera B, Widom C. Childhood victimization and violent offending. Violence Vict 1990;5(1):19-35.

10. Rivara R, Gurney J, Ries R, et al. A descriptive study of trauma, alcohol, and alcoholism in young adults. I Adolesc Health 1992;13:663-67.

11. Wolfe D, Jaffe P, Wilson S, Zak L. A multivariate investigation of children's adjustment to family violence. In: Hotaling G, Finkelhor D, Kirkpatrick J, Strauss M (Eds.), Family Abuse and its Consequences: New Directions in Research. Newbury Park, CA: Sage Publications, 1988.

12. Sturkie K, Flanzer J. Depression and self-esteem in the families of maltreated adolescents. Soc Work 1987;32(6):491-96.

13. Suh E, Abel E. The impact of spousal violence on the children of the abused. J Ind Soc Work 1990;4(4):27-34.

14. Hershorn M, Rosenbaum A. Children of marital violence: A closer look at the unintended victims. Am J Orthopsychiatry 1985;55(2):260-66.

15. Lorion RP, Saltzman W. Community Experience Survey. University of Maryland, College Park, MD, 1991.

16. Derogatis LR. The Brief Symptom Inventory (BSI): Administration, Scoring \& Procedures Manual - II. Clinical Psychometric Research, 1992.

17. Kovacs M. Children Depression Inventory: Manual. Multi-Health Systems, 1992.

18. Hudson WW. Index of Self-Esteem (ISE). Tempe, AZ: WALMYR Publishing, 1992.

Received: January 7, 1999

Accepted: April 16, 1999

\section{O M I N G E V E N T S A C T I VITÉS À VEN I R}

To be assured of publication in the next issue, announcements should be received by July 15, 1999 and valid as of August 30, 1999 . Announcements received after July 15, 1999 will be inserted as time and space permit.

Pour être publiés dans le prochain numéro, les avis doivent parvenir à la rédaction avant le 15 juillet 1999 et être valables à compter du 30 août 1999. Les avis reçus après le 15 juillet 1999 seront insérés si le temps et l'espace le permettent.

40th Annual Institute on Addiction Studies

Celebrating the Journey

11-15 July 1999

Contact:

Nancy Bradshaw or Sandra Caswell

Concerns Canada

Tel: 416-293-3400Ｅ-mail: concerns@sympatico.ca

\section{AIDS IMPACT}

Biopsychosocial Aspects of HIV Infection

4th International Conference

Connecting a World of Resources

15-18 July 1999

Ottawa, ON

Hosted by the Canadian Psychological Association (CPA)

AIDS IMPACT provides a multidisciplinary forum where

researchers, care providers, consumers and others can

address changing trends in the HIV epidemic. The confer-

ence aims to bring research to practice by delivering exper-

tise and skills to the front lines.

Contact:

AIDS Impact 1999 Secretariat

Tel: 613-237-2144

Fax: 613-825-0530

Web site: www.aidsimpact.com

World Conference on Breast Cancer

An international and multi-disciplinary forum where all the issues around breast cancer can be addressed.

26-31 July 1999

Contact:

Ottawa, Ontario

World Conference on Breast Cance

Tel: 613- 549-1118

E-mail: brcancer@kos.net

Fax: 613-549-1146

Ageing in a Society for All Ages

4th Global Conference of the International Federation on

Ageing

5-9 September 1999

Montréal, QC

Contact:

Tel: 514-287-9898, ext. 259

E-mail: ageingconf@jpdl.com

Fax: 514-287-1248

Mediation: Reaching Families and Beyond

13th Annual National Conference of Family Mediation

Canada co-hosted with The Ontario Association for Family Mediation

20-24 October 1999

Contact:

Family Mediation Canada

Tel: 519-585-3118

Toronto, ON

Fifth International Conference on

Children Exposed to Family Violence: Creating a Legacy of

Hope

Co-hosted by BC/Yukon Society of Transition Houses and the London Family Court Clinic

27-30 October 1999 Vancouver, BC

Contact:

BC/Yukon Society of Transition Houses

Tel: 604-669-6943

Fax: 604-682-6962

E-mail: hdempst@istar.

http://home.istar.ca/ bcysth

The Dawn of a New Era: Challenges for Baby Boomers

Canadian Association on Gerontology Annual Scientific and Educational Meeting

4-7 November 1999

Ottawa, ON

Contact:

CAG Conference Secretariat

Tel: 613-728-9347

E-mail: cagacg@magi.com

Fax: 613-728-8913

The Early Years: Supporting Families \& Young Children Sponsored by Interprofessional Continuing

Education, The University of British Columbia

27-29 January 2000

Vancouver, BC

Contact:

The Early Years

Continuing Education in the Health Sciences

The University of British Columbia

Tel: 604-822-4965

Fax: 604-822-4835

E-mail: elaine@cehs.ubc.ca

Éditorial, suite de la page 180

également nécessaires aux organismes bénévoles pour défendre l'élaboration de leurs politiques auprès du public et des décideurs.

Pour relever les défis posés à l'amélioration de la surveillance de la santé au Canada, il sera important d'avoir des objectifs précis et de travailler en collaboration. Avoir des objectifs précis est nécessaire pour affecter les ressources limitées aux questions d'importance nationale s'agissant de la santé publique et aux besoins des principaux utilisateurs des données produites par la surveillance de la santé. La collaboration est indispensable si l'on veut améliorer et partager de façon efficace et efficiente les données sur la santé. Espérons que nous tous, qui travaillons dans le domaine de la surveillance de la santé publique, saurons relever ce défi.

\section{REFERENCES/BIBLIOGRAPHIE}

1. Institute of Medicine. The Future of Public Health. National Academy Press, 1988; 240 pp (ISBN 0-309-03830-8).

2. Federal/Provincial/Territorial Surveillance Integration Design Team. A Discussion Paper on an Integrated National Health Surveillance Network for Canada. Ottawa: Health Canada, 1998; 40 pp.

3. Krever Commission Report. Ottawa, Public Works and Government Services Canada, 1997, 1134 pp (http://www.hc-sc.gc.ca/english/krever/index.html). 